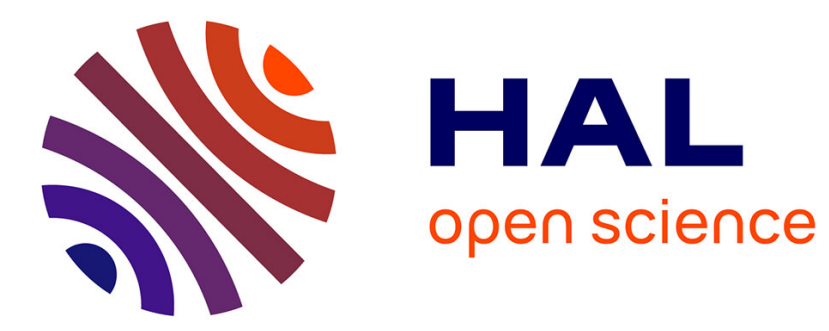

\title{
ANTENNE ULTRASONORE À FOCALISATION
}

\author{
M.-F. Dandine, R. Barriol
}

\section{To cite this version:}

M.-F. Dandine, R. Barriol. ANTENnE ULTRASOnORE À FOCALISATION. Journal de Physique

IV Proceedings, 1992, 02 (C1), pp.C1-919-C1-922. 10.1051/jp4:19921201 . jpa-00251167

\section{HAL Id: jpa-00251167 https://hal.science/jpa-00251167}

Submitted on 1 Jan 1992

HAL is a multi-disciplinary open access archive for the deposit and dissemination of scientific research documents, whether they are published or not. The documents may come from teaching and research institutions in France or abroad, or from public or private research centers.
L'archive ouverte pluridisciplinaire HAL, est destinée au dépôt et à la diffusion de documents scientifiques de niveau recherche, publiés ou non, émanant des établissements d'enseignement et de recherche français ou étrangers, des laboratoires publics ou privés. 


\title{
ANTENNE ULTRASONORE À FOCALISATION
}

\author{
M.-F. DANDINE et R. BARRIOL* \\ E.N.S.A.M., 2 Bd Ronceray, BP. 3525, F-49035 Angers, France \\ ${ }^{*}$ L.M.A.I., Université de Perpignan, Av. de Villeneuve, F-66860 Perpignan, France
}

\begin{abstract}
A theorotical study about the operating optimization of a focusing submarine array prototype allowed us to get better results but also establish a general rule of operating and have a geometrical apprehension of it. Later on, the carrying out of the geometrical optimization resulted in a new prototype, which proved to be very performing in the field of axial and lateral responses. Finally, a study of similarity enebled us to build a homolos gous model in the aerial field; the experimentation confirmed the key points of the theory.
\end{abstract}

1. DESCRIPTION DU PROTOTYPE.

Le prototype construit et mis au point dans nos laboratoires est de géométrie et de fonctionnement similaires à celui réalisé par SHIBATTA (1). La différence réside dans le fait que celui-ci possède une couronne supplémentaire pour le fonctionnement en émission.

L'étude présentée a été effectuée dans le cadre d'un programme de recherche qui visait à la reconnaissance des objets enfouis dans les sédiments marins (2). Le principe d'antennerie s'imposait; traitement focal en employant l'échographie en mode $C$ pour reproduire l'image (3).

L'antenne comporte, disposés dans un rême plan, sur quatre couronnes concentriques, de rayons respectifs: 0,$125 ; 0,180 ; 0,300$ et 0,500 m, et selon huit rayons équirépartis:

- 32 transducteurs pour l'émission,

- 24 transducteurs pour la réception (sur les trois couronnes extérieures).

La fréquence de fonctionnement retenue est $46 \mathrm{KHz}$; la focalisation est obtenue par commande des phases $\varphi_{i}$ des différentes couronnes par un micro-ordinateur.

Les chercheurs du LMAI (2) ont, dans un premier temps déterminé les déphasages à partir d'un modèle géométrique (M.I), mais du fait de son manque de réalité physique, il $n ' a$ pas été obtenu de résultat:significatif. Ensuite a été établi un modèle d'acoustique physique (M.II), où les déphasages ont été déterminés ponctuellement par balayage informatique; des résultats corrects ont été obtenus dans le cas d'un seul milieu, mais les expériences avec passage d'un interface eau-sédiment n'ont pas donné jusqu'à présent de résultats.

Pour toutes ces raisons, il s'est avéré nécessaire d'étudier l'optimisation de fonctionnement de ce prototype, ainsi que son optimisation géométrique (4).

\section{OPTIMISATION DE FONCTIONNEMENT.}

Les lois de l'acoustique linéaire appliquées à la géométrie de l'antenne impliquent que la modulation de pression sur l'axe de l'antenne soit fonction des:

- Nivequx d'émission A des transducteurs, 
- Phases relatives $\varphi$ des différentes couronnes (indicées i),

- Rayons a des couronnes,

- Distancesrrd'un transducteur à unpoint de l'axe de l'antenne.

Dans un premier temps, 1'étude effectuée dens un milieu honogène isotrope et en émission conduit, pour le carré de ${ }^{1}$ amplitude de modulation de pression $P_{x}$ à:
\[ P_{x}^{2}=\left(\sum_{i} A_{i} \cdot r_{i}^{-1} \cdot \cos \alpha_{i}^{2}+\left(\sum_{i} A_{i} \cdot r_{i}^{-1} \cdot \sin \alpha_{i}^{2} \text { avec: } \alpha_{i}=k \cdot r_{i}-\varphi_{i}\right.\right. \]

Le calcul analytique d'optimisation aboutit aux conditions suivantes:

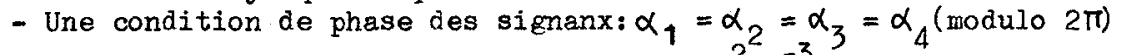

- Une relation de géométrie de l'antenne: $a_{i}^{2} \cdot r_{i}^{-3}=$ const. Cette dernière relation renseigne sur les dimensions optimales.

Un programie de simulation sur micro-ordinateur (M.IV) permet de constater que la focalisation est effectivement obtenue, mais décalée vers le centre de l'antenne par rapport au point visé. Cet écart $\Delta x$ est une fonction croissante de la distance du point visé au centre de l'antenne (Fig. No1). Ce décalage a pu être compensé par une fonction de correction établie par comparaison des dorsales avec celles déterminées à partir de la théorie des ondes planes (M.III) et possédant les mêmes caractéristiques théoriques, pour lequel la focalisation avait effectivement lieu au point visé:

$$
\Delta x=P(x) \cdot x^{-1} \cdot(P(x))^{-1}
$$
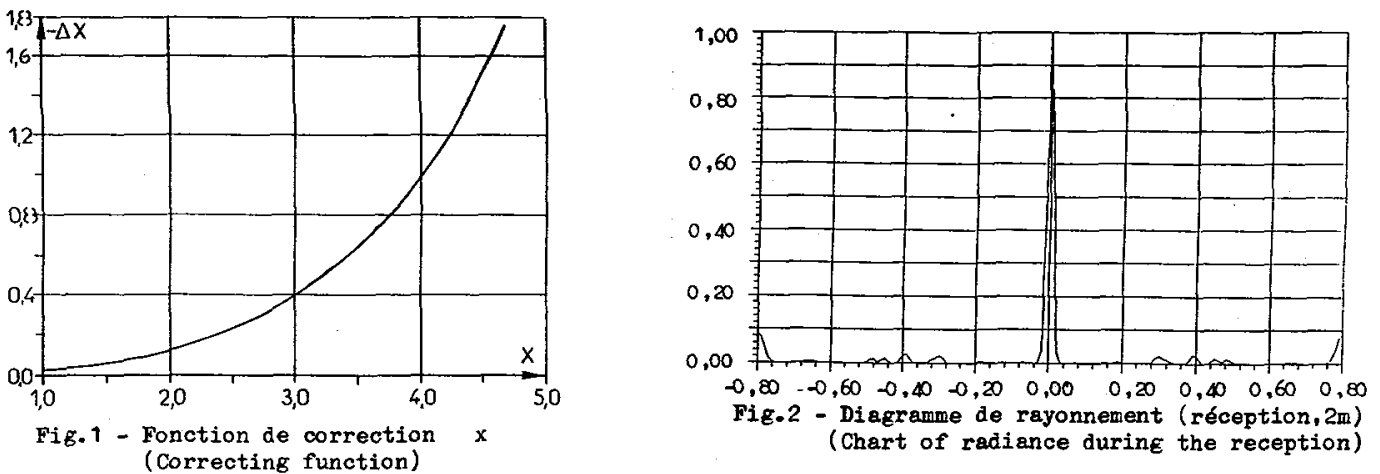

La longueur $\Delta l$ de la tâche focale a été évaluée à un niveau de -3d̉ , et comparée à la longueur $\Delta \mathrm{l}^{\prime}$ correspondant aux conditions de fonctionnement non optimisées:

$\begin{array}{rccc}\mathrm{x},(\mathrm{m}) & 2 & 3 & 4 \\ \Delta 1,(\mathrm{~m}) & 0,76 & 1,71 & 3,06 \\ \Delta 1 ;(\mathrm{m}) & 0,83 & 1,83 & 3,16\end{array}$

L'étude des diagrammes de rayonnement a été effectuée en vue de caractériser la directivité:

- Le demi-angle d'ouverture est de $1,14^{\circ}$ pour une distance de focslisation de $2 \mathrm{~m}$, - Le niveau relatif des lobes secondaires est de -20 dB; les gains obtenus sont triès nets par rapport à ceux de la structure non optinisée $(-11 d B)$.

Une étude en réception dans un nême milieu, en appliquant un calcul sinilaire sur le produit des signaux, conduit égglement à 1'optinisation (Fig.N०2): La loneueur de la tâche focale à $-3 \mathrm{~dB}$ est de $0,30 \mathrm{n}$, le deni-anele d'ouverture du lobe principal $0,3^{\circ}$, le niveau des lobes secondaires passe de $-25 \mathrm{~dB}$ à $-40 \mathrm{~dB}$.

L'utilisation sinultanée en érission et en réception pernet d'aboutir à un fonctionnement optinisé très performant. La tâche focale est considérablement affinée et l'apodisation très bonne, puisque les lobes secondaires sont d'un niveau de -godB. Le deni-angle d'ouverture est difficilement décelable à -3dB (avec cependant une très bonne précision sur le pas de calcul). 


\section{3: OPTIMISATION GEOMETRIQUE.}

L'optimisation de fonctionnement étant réalisée, il restait à déterminer si, pour une envergure $d$ 'antenne inchangée, les valeurs des rayons des couronnes correspondaient à des performances optimales.

Tenant compte du fait qu'aucune loi mathématique ne régissait la structure actuelle, un modèle informatique par balayage des rayons des couronnes intermédiaires a été établi. Ceci a permis de mettre en évidence que la meilleure résolution longitudinale correspondait à des veleurs des rayons intermédiaires très proches de celles des rayons extrêmes (Fig.N.3), et conduit à un nouveau prototype comportant seulement deux couronnes. Celui-ci est piloté avec la même loi de phase que le précédent, et la fonction de correction est également applicable.

Les résultats obtenus en émission sont très prometteurs:

- L'amélioration de la résolution axiale peut atteindre $46 \%$ (Fig.N 4 ),

- Le lobe principal de rayonnement a un demi-engle d'ouverture de seulement $0,6^{\circ}$; mais il existe une perte de $6 \mathrm{~dB}$ sur les lobes secondaires (ce résultat est cependant meilleur que pour le cas de la structure non optimisée).

Comme pour le prototype initial, le traitement en réception permettra une amélioration considérable de la définition de la tâche focale, ainsi que l'apoóisation.

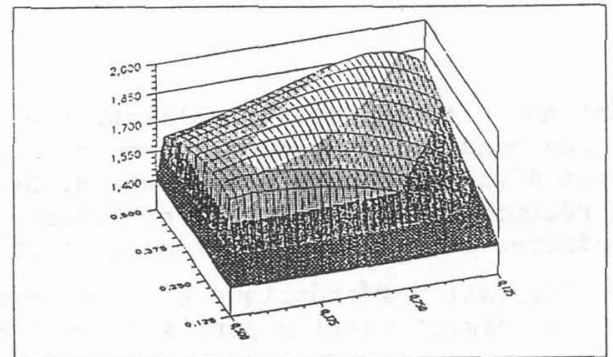

Fig. 3 - Longueur de la tiche focale en fonction des rayong des couronnes intermódiaires. (Length of the focal apot in accordance with the radil of intermediary rings)

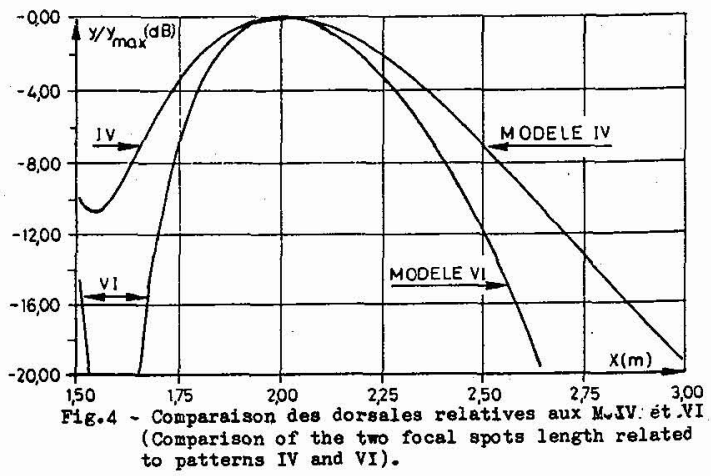

4. REMARQUES, EFFICACITE ENERGETIQUE.

Dans le but d'obtenir un maximum élevé de pression au point de focalisation,piuaieurs configurations d'émission et de réception ont été testées:

- Niveaux d'émission identiques pour les différentes couronnes,

- Niveaux de modulation de pression provenant des différentes couronnes identiques dans le domaine de focalisation.

Il $n^{\prime a}$ pas été constaté de différences sensibles dans la longueur de la tâche focale, par contre, dans le second cas, le niveau de modulation de pression est plus élevé.

Les performances ont été caractérisées par la proposition d'un facteur énergétique adimentionnel qui permet la comparaison quantitative entre l'énergie acoustique locale globale correspondant aux phénomènes d'interférences acoustiques et la somme des énergies acoustiques locales provenant individuellement des différentes sources. Par la suite, le facteur énergétique moyen par couronne été introduit,il correspond à la contribution moyenne d'une couronne dans le bilan énergétique local:

$$
n=\mathrm{P}^{2} \cdot\left(\mathrm{n} \cdot \sum_{\mathrm{n}} \mathrm{p}_{i}^{2}\right)^{-1} ; \mathrm{n}=\text { nombre de couronnes. }
$$

Ce facteur permet une comparaison d'efficacités locales entre des structures différentes, et la mise en évidence des avantages apportés par l'optimisation de fonctionnement et par la nouvelle structure de prototype (U.VI) par rapport au prototype antGrieur, même de fonctionnement optimisé (M.IV); augmentation de $24 \%$.

Il est permis alors de s'interroger sur la nécessité, dans des cas particuliers, de corriger la défocalisation; en effet, sans correction, l'énergie locale est toujours maximale au point visé. L'intérêt, dans certains cas, sera de chercher un 
compromis entre ces deux modes de fonctionnement, ou bien'de favoriser l'un per rapport à l'autre.

\section{EXPERIMENTATION.}

Dans un premier temps, 1 'expérimentation a été réalisée sur maquette; le passage du prototype sous-marin à 1 'antenne homologue aérienne a pu se faire par des calculs de similitude. L'envergure est de $2,12 \mathrm{~m}$ et $\mathrm{la}$ fréquence de fonctionnement de $5 \mathrm{KHz}$. Les mesures effectuées pour une distance de focalisation de 4,40m ont donné des résultats probants, ì savoir:

* Dans le cas de la configuration initiale:

- La fonction de correction a été vérifiée,

- L'optimisation apportée sur la longueur de la thche focale également,

- Le diagramme de rayonnement caractérisé par un lobe principal de $1,4^{\circ}$ et un niveau de lobe secondaire de $-10 \mathrm{~dB}$; cette différence par rapport à le théorie s'explique par les conditions extérieures d'expérimentation (confinement),

- La vérification, avec les conditions optimisées, de l'augmentation du facteur $\eta$,

* Pour un fonctionnement avec deux couronnes:

- L'amélioration supplémentaire du facteur énergétique,

- La longueur de la tâche focale ainsi que le décalage du point de focalisation sont en bon accord avec le mođèle thérique,

- Le lobe principal a un demi-angle d'ouverture d'environ $1^{\circ}$.

6. CONCLUSION.

L'étude d'optimisation de fonctionnement de l'antenne ultrasonore a focalisation a permis d'établir une relation sur sa géométrie. Les conaitions de fonctionnement optimales ont été définies grêce à l'établissement d'une fonction de correction. De plus l'utilisation simultanée en émission et en réception donne dans ces conditions une tâche focale très affinée et des lobes secondeires de très bas niveaux relatifs.

Le nouveau prototype obtenu à l'aide de l'optimisation géométrique est très performant, et la proposition d'un facteur énergétique adimentionnel a permis de confirmer 1a qualité des optimisations.

Les points forts de la thérie ont jusqu'à présent été testés avec succés sur une maquette homologue aérienne.

Les études se poursuivent actuellement dans différentes directions: - Remplacement des déphasages par des retards, car le régime pulsé s'impose dans ces conditions; les résultats fondamentaux demeurant inchangés. Ces retards seront déterminés grâce à la loi de phase démontrée, qui est une loi générale.

- L'étude se poursuit également pour l'application aux milieux stratifiés,

- La poursuite de l'expérimentation dans le domaine sous-marin ainsi que sur maquette homologue est prévue.

\section{REFERENCES.}

(1) - S.SHIBATA, T.KODA, S.MATSUMOTO and J.YAMAGA. COAXIAL CIRCULAR SPHERICAL ARRAY FOR ULTRASONIC IMAGING. J.Acoust. Soc. Am. Vol. 62, No. 4, Oct.1977, p 819-824.

(2) - J.BRESSON, R.BARRIOL et J.P.LONGUEMARD.

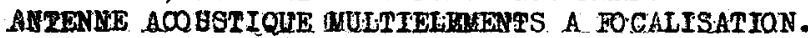
Revue Physique Appliquée T.22, 0ct.1987, p 1177-1184.

(3)- S.SHIBATA, T.KODA and J.YAMAGA. C-MODE ULTRASONIC IMAGING. Ultrasonics, Mars 1978, p65-68.

(4) - M-F.DANDINE. OPTIMISATION D'UNE ANTENNE AOOUSTIQUE MULTI ELEMENTS A FOCALISATION. Mémoire Ingénieur CNAM, PARIS, Nov. 1990. 